\title{
A Strained Partnership: Krise und Resilienz in den transatlantischen Beziehungen 20 Jahre nach 9/11
}

\author{
Florian Böller
}

Angenommen: 9. November 2021 / Online publiziert: 29. November 2021

(C) Der/die Autor(en) 2021

2021 lieferte aus transatlantischer Perspektive gleich mehrere Zäsuren. Im Januar wurde US-Präsident Donald Trump, dessen disruptive Politik diverse Konflikte mit Europa provozierte, durch Joseph R. Biden abgelöst. Im August endete in Afghanistan der längste Einsatz in der Geschichte der NATO mit einem chaotischen Abzug und der Machtübernahme der Taliban, fast 20 Jahre nach dem Beginn des Krieges. Und schließlich läuteten die Bundestagswahlen im September das Ende der Amtszeit Angela Merkels ein, die als Bundeskanzlerin in 16 Regierungsjahren auf vier US-Präsidenten traf. Diese Zäsuren bieten Anlass genug, eine Bilanz der transatlantischen Beziehungen seit $9 / 11$ zu ziehen.

In den vergangenen 20 Jahren wurden die transatlantischen Beziehungen durch eine Reihe außenpolitischer Krisensituationen, externe Schocks und neue internationale Konflikte herausgefordert. Für die traditionelle Führungsmacht der „Sicherheitsgemeinschaft" (Deutsch et al. 1957), die USA, aber auch für Deutschland ergaben sich daraus neue Anforderungen - sowohl mit Blick auf externe Handlungszwänge und Bedrohungen als auch innerhalb der Allianz, in der teils inkompatible Erwartungen gegenüber den jeweiligen Partnern aufeinandertrafen. Gleichzeitig ist Vorsicht in Anbetracht der inflationären Verwendung des Krisenbegriffs im öffentlichen (und akademischen) Diskurs geboten. Nicht jede außenpolitische Krisensituation löst gleichzeitig auch eine transatlantische Krise aus. Internationale Konflikte wie die Kriege in Afghanistan, Irak, Libyen und Syrien, die russische Annexion der Krim oder der Bürgerkrieg im Jemen offenbaren Differenzen zwischen den Mitgliedern der Sicherheitsgemeinschaft. Auseinandersetzungen über konkrete politische

\footnotetext{
Florian Böller $(\bowtie)$

Fachbereich Sozialwissenschaften, Arbeitsbereich Internationale Beziehungen/Außenpolitik,

Technische Universität Kaiserslautern, Erwin-Schroedinger-Str., Gebäude 57,

Raum 482, 3049, 67663 Kaiserslautern, Deutschland

E-Mail: boeller@sowi.uni-kl.de
} 
Projekte, wie etwa das Nord Stream 2 Pipeline-Projekt führen aufgrund nicht-deckungsgleicher wirtschafts- und sicherheitspolitischer Interessen zu deutlichen Disputen. Solche Kontroversen gehören aber regelmäßig zum Aushandlungsprozess der transatlantischen Akteure, schließlich ist diese Sicherheitsgemeinschaft ,pluralistisch“ aufgrund national geprägter Interessen und Normen (Deutsch et al. 1957). Daher überschreitet ein Disput innerhalb der Sicherheitsgemeinschaft die Schwelle zu einer Krise aus meiner Sicht nur dann, wenn grundlegende Werte und Prinzipien der Gemeinschaft gefährdet sind. Dazu zählen neben den Grundnormen der kollektiven Verteidigung und des Multilateralismus auch das Vertrauen zwischen Bündnispartnern (Koschut 2016). Gerät einer dieser drei Grundpfeiler ins Wanken, dann ist tatsächlich von einer Krise im transatlantischen Verhältnis zu sprechen. Im Folgenden werde ich die transatlantischen Beziehungen nach 9/11 anhand dieses Maßstabs untersuchen und das Augenmerk auf Krisen und „beinahe Krisen“ richten, also jene Fälle, in denen die Akteure eine krisenhafte Situation durch gemeinsame Aushandlungsprozesse auffangen konnten. Der Mehrwert dieser Analyse liegt in der Identifizierung von Triebkräften der Krisen auf der einen Seite und auf der anderen Seite von Mechanismen, die zur Resilienz der Beziehungen beitrugen. Wie ich zeigen werde, übertraten nur in zwei Situationen der vergangenen 20 Jahre die transatlantischen Beziehungen die Schwelle zur Krise, nämlich in der Folge der Kontroverse um den Irakkrieg 2002/2003 und während der Präsidentschaft Trumps. Was bei der Betrachtung dieser Fälle in analytischer Perspektive deutlich wird, ist die Bedeutung innenpolitischer Faktoren bei der Entstehung allianzinterner Konflikte. Externe Schocks stellten die Verbündeten vor neue Herausforderungen und auch langfristig wirkende Entwicklungen wie der Dauerkonflikt in Afghanistan oder der Aufstieg Chinas stellten den bündnisinternen Zusammenhalt auf die Probe. Doch es waren nicht allein neue externe Herausforderungen, die das Ausmaß und die Virulenz der Krisen bestimmten. Vielmehr wurden die Krisen durch innenpolitische Anreizstrukturen, gesellschaftliche Konfliktlinien und nationale Präferenzen maßgeblich beeinflusst. Dieses Argument, das domestischen Faktoren analytischen Vorrang vor systemischen Konstellationen einräumt, werde ich im Folgenden für die transatlantische Sicherheitsgemeinschaft nach 9/11 exemplarisch darstellen.

\section{Preventive War: Transatlantische Krise in Folge der Irakkriegsentscheidung}

Die Anschläge des 11. Septembers führten die Sicherheitsgemeinschaft nicht unmittelbar in eine Krise. Vielmehr bekundeten die Bündnispartner rasch ihre Solidarität mit den USA. Bereits am Tag nach den Anschlägen erklärte Bundeskanzler Gerhard Schröder Deutschlands ,uneingeschränkte Solidarität“ und auf NATO-Ebene wurde zum ersten Mal überhaupt der Bündnisfall nach Artikel 5 des Washingtoner Vertrags ausgerufen. Die kurz darauf beginnende Afghanistanintervention wurde zwar militärisch primär von US-Streitkräften durchgeführt, Truppenentsendungen anderer NATO-Staaten signalisierten jedoch die Handlungsfähigkeit des Bündnisses im Kampf gegen den internationalen Terrorismus, der als Bedrohung der gesamten westlichen Zivilisation aufgefasst wurde. Als sich der Fokus der Bush-Administra- 
tion auf den Irak zu richten begann, erodierte die „uneingeschränkte Solidarität“ allerdings schnell. Das hatte vor allem drei Gründe: Erstens waren die Regierungen insbesondere in Frankreich und Deutschland (wie auch mehrheitlich die europäische Öffentlichkeit) nicht von der zentralen Legitimierung des Kriegs, der Existenz irakischer Massenvernichtungswaffen, überzeugt. In den USA dagegen sicherte sich Präsident Bush nicht nur die Zustimmung der Legislative, inklusive einiger Stimmen der Demokratischen Opposition, vielmehr befürwortete auch die US-Öffentlichkeit mehrheitlich einen Präventivkrieg gegen den Irak - wohlgemerkt vor dem Hintergrund von Falschinformationen. Zweitens verstießen die USA in ihrem unilateralen und den VN-Sicherheitsrat letztlich ignorierenden Vorgehen gegen völkerrechtliche Prinzipien, die von anderen Mitgliedern der Sicherheitsgemeinschaft höher gewichtet wurden. Hinzu kommt in Deutschland drittens die Politisierung der Irakkriegsentscheidung während des Wahlkampfs im Sommer 2002 durch die Schröder-Regierung (Forsberg 2005). Ohne dass eine deutsche Beteiligung am Krieg von den USA in Erwägung gezogen worden wäre, machte Bundeskanzler Schröder sein entschiedenes Nein gegen den Krieg zu einem zentralen Wahlkampfthema. Im Verbund mit Frankreich und Russland - mithin entgegen der traditionellen Bündnisorientierung Deutschlands - wurde auch die Bühne des VN-Sicherheitsrats genutzt, um gegen die als hegemonial wahrgenommene Politik der USA zu opponieren.

Auf Ebene der transatlantischen Allianz eskalierte diese Auseinandersetzung über Interessen (Sicherheitsinteressen der USA) und Werte (Betonung völkerrechtlicher Normen) zu einem Eklat als Deutschland, Frankreich und Belgien im Vorfeld des Irakkriegs im NATO-Rat die Entscheidung über die Autorisierung von Verteidigungsplanungen für die Türkei (zum Schutz vor einem möglichen irakischen Angriff) im Februar 2003 blockierten. Beobachter sprachen in diesem Zusammenhang von einer „Nahtod-Erfahrung“ der NATO (Terriff 2004), da hier eine der zentralen Bündnisnormen, nämlich das Prinzip der kollektiven Verteidigung, in Frage gestellt wurde. Letztlich konnte der offene Bruch im Bündnis vermieden werden, gleichwohl zeigt diese Krise, wie divergente Interessenlagen durch innenpolitische Kalküle aufgeladen werden können und in der Krisendynamik nicht-intendierte Folgen - hier der Konflikt im NATO-Rat - den Zusammenhalt im Bündnis gefährden.

\section{2 ,Nation-building at Home“: Obamas Wandel und der transatlantische Burden-Sharing Konflikt}

In klarer Abgrenzung zu seinem Amtsvorgänger betonte Barack Obama die Bedeutung multilateraler Zusammenarbeit und bemühte sich um die Re-Legitimierung der US-Weltmachtrolle. Obamas Kurswechsel in der US-Außenpolitik hatte jedoch einen paradoxen Effekt. Einerseits trug er dazu bei, die Gräben zwischen den USA und Europa hinsichtlich zentraler Grundprinzipien der Allianz zu überbrücken und verlorengegangenes Vertrauen, gerade auch gegenüber der transatlantischen Öffentlichkeit wiederherzustellen. ${ }^{1}$ Auf der anderen Seite provozierte Obamas außen- und

\footnotetext{
1 Zumindest bis zum Beginn der NSA-Affäre 2016, die jedoch nie einen Krisenstatus im Sinne der oben eingeführten Definition erreichte (Schünemann et al. 2018).
} 
sicherheitspolitische Strategie das Aufbrechen einer alten Konfliktlinie: der Frage fairer Lastenteilung im Bündnis. Für diese Dynamik waren innenpolitische Faktoren entscheidend. Obamas Wahlsieg basierte zu nicht unerheblichen Teilen auf der Frustration der amerikanischen Wählerschaft mit dem kosten- und verlustreichen Irakkrieg. Der Präsident erkannte - zumal seit Amtsantritt mit der Bewältigung der Great Recession konfrontiert - dass die Gesellschaft eine Fokussierung sozialer und wirtschaftlicher Herausforderungen einforderte. Aus der Prämisse des ,nation-building at home“ (White House 2011) folgten neue Erwartungen an die Bündnispartner. In dem Maße die USA ihren Fokus nach innen richteten, forderten sie von ihren Alliierten sich stärker an der Stabilisierung der liberalen Weltordnung zu beteiligen (Böller und Hagemann 2017).

Der erste Testfall dieser neuen Doktrin während des Libyenkonflikts 2011 führte sogleich zu einem transatlantischen Disput als sich zeigte, dass die europäischen Partner keineswegs in der Lage (und im Falle Deutschlands willens) waren, militärisch eine führende Rolle zu übernehmen. Die USA brachten ihre Frustration über die angesichts mangelnder Verteidigungsausgaben eingeschränkte Kooperationsfähigkeit auf europäischer Seite deutlich zum Ausdruck (Gates 2011). Streit über eine faire Lastenteilung war auch in der Phase des Ost-West-Konflikts Teil des allianzinternen Konfliktmanagements. Die Salienz des Konflikts wurde in dieser Phase jedoch durch eine größere Kostensensitivität auf Seiten der Führungsmacht erhöht, während andererseits die europäischen Partner in Anbetracht eigener wirtschaftspolitischer Krisenlagen die Anhebung der Verteidigungshaushalte nicht umsetzen konnten. Ein Umdenken setzte in dieser Hinsicht erst mit der Ukrainekrise 2014 ein, da hier die Verletzlichkeit der europäischen Sicherheitsarchitektur augenfällig wurde. Dies führte auf NATO-Ebene zur Stärkung der kollektiven Verteidigung und in den Mitgliedsstaaten zu teils deutlichen Erhöhungen der Verteidigungsausgaben (Haesebrouck 2021). Insofern bewirkte die Ukrainekrise ein Zusammenrücken der Bündnispartner, was auch an der Aufrechterhaltung des Sanktionsregimes gegenüber Russland erkennbar wurde.

Der Libyenkonflikt wurde letztlich eingehegt, obwohl die deutsche Enthaltung bei der Abstimmung über die Legitimierung der Intervention durch den VN-Sicherheitsrat im März 2011 für erhebliche Verstimmungen bei den Partnern gesorgt hatte. Deutschland stellte sich mit dieser Enthaltung an die Seite Russlands und Chinas, was insbesondere von Frankreich, Großbritannien und den USA als Affront wahrgenommen wurde (Koschut 2014). Wiederum spielten innenpolitische Erwägungen eine wichtige Rolle für die außenpolitische Positionierung. Die Entscheidung über die Enthaltung wird auf wahltaktische Erwägungen des damaligen Außenministers Guido Westerwelle zurückgeführt, vor dem Hintergrund der Landtagswahlen in BadenWürttemberg (kritisch Hansel und Oppermann 2016). Dass diese Konfliktsituation nicht in eine transatlantische Krise (im Sinne der oben definierten Schwelle) eskalierte, lässt sich einerseits auf das demonstrative Eingeständnis von Bedauern über die Folgen der Libyenentscheidung durch die Bundeskanzlerin und andererseits auf symbolische Kompensationen Deutschlands gegenüber den Verbündeten (Erhöhung der Kontingente für die NATO-Luftraumüberwachung in Afghanistan) zurückführen (Koschut 2014, S. 554) - womit letztlich die eingeübten institutionellen und multilateralen Konfliktlösungsmechanismen aktiviert wurden. 


\section{3 „America First“": Vertrauenskrise der transatlantischen Beziehungen in der Ära Trump}

Mit dem Beginn der Trump-Präsidentschaft endete die Phase relativer Stabilität, die sich in Folge der Ukrainekrise innerhalb der Sicherheitsgemeinschaft etabliert hatte. Bereits nach wenigen Wochen im Amt war klar, dass sich Trumps nationalistischer „America First“-Kurs auch gegen die traditionellen Verbündeten richten würde. Tatsächlich durfte diese Ausrichtung nicht überraschen, war doch die Kritik an der fehlgeleiteten Außenpolitik seiner Amtsvorgänger ein wichtiges Argument im Wahlkampf Trumps. Die Eliten in Washington hätten sich von internationalen Partnern über den Tisch ziehen lassen und sich nicht auf die Realisierung nationaler Interessen konzentriert, sondern unnötige Projekte wie Demokratieförderung und humanitäre Interventionen verfolgt (New York Times 2016; Junker 2021, S. 293. In dieser Hinsicht ähnelte Trumps Argument Obamas ,nation-building at home“, wenngleich die präferierte Lösung nicht in multilateraler Lastenteilung, sondern in der unilateralen Verfolgung eng gefasster nationaler Interessen bestand. Der Ausgangspunkt dieser transatlantischen Krise lag also zentral in der Unzufriedenheit maßgeblicher Teile der US-Wählerschaft mit den ausbleibenden Profiten der liberalen Weltordnung (Tuschhoff 2021).

Die transatlantische Partnerschaft geriet vor allem aus zwei Gründen in eine Krise: Zum einen, da Trumps Politik ein zentrales Bündnisprinzip untergrub, nämlich die kollektive Verteidigung, und zum anderen durch die Präferenz für unilaterale Strategien. Beide Aspekte griffen die Vertrauensbasis der Sicherheitsgemeinschaft an, was die Virulenz der Krise verschärfte.

Beim NATO-Gipfeltreffen in Brüssel 2018 eskalierte Trump rhetorisch die Debatte um Verteidigungsausgaben, indem er während einer Sitzung des NATO-Rats mit dem Austritt der USA drohte (Schuette 2021). In einem Fernsehinterview kurz danach stellte Trump zudem in Frage, inwiefern die USA im Bündnisfall Truppen zur Verteidigung von Montenegro entsenden sollten (Factaba.se 2018). Diese Aussagen gepaart mit der offenen Drohung eines Austritts der USA säten in erheblichen Maße Misstrauen unter den Verbündeten. Auch wenn die USA unter Präsident Trump ihre materiellen Beiträge zur kollektiven Verteidigung im Rahmen der European Deterrence Initiative sogar noch steigerten und der Versuch eines Austritts aus der Organisation vom Kongress möglicherweise blockiert worden wäre, ließ Trumps Vorgehen die Vertrauensbasis der Sicherheitsgemeinschaft erodieren. Schließlich beruht die Bündnisnorm nach Artikel 5 des NATO-Vertrags in erster Linie auf der diffusen Erwartung von Reziprozität, da es einen Automatismus zu substanzieller (militärischer) Hilfe nicht gibt. Trumps transaktionales Verständnis der NATO widersprach diesem vertrauensbasierten Prinzip fundamental.

Hinzu kommt, dass Trump auf einer Vielzahl von Politikfeldern - in der Handels-, Klima-, Rüstungskontroll-, und Nichtverbreitungspolitik - reihenweise internationale Abkommen aufkündigte, und damit die Erwartungssicherheit über künftiges Handeln und die Zuverlässigkeit der USA beschädigte. Hierzu zählten mit den INFund Open Skies-Verträgen auch Abkommen, die primär für die europäische nukleare Ordnung relevant waren. 
Der Vertrauensverlust in Folge der Politik Trumps zeigte sich nicht nur in öffentlichen Umfragen (z.B. Politbarometer 2018) und entsprechenden Aussagen europäischer Verbündeter, etwa Frankreichs Präsident Emmanuel Macron, der einen „Hirntod“ der NATO diagnostizierte (Reuters 2019). Insbesondere Frankreich und Deutschland reagierten vielmehr auch mit symbolischen Schritten in Richtung einer größeren Unabhängigkeit Europas von den USA. Beispiele hierfür sind die PESCOInitiative innerhalb der Europäischen Union oder der Vertrag von Aachen 2019, mit dem Deutschland und Frankreich ihren Willen zu engerer sicherheitspolitischer Kooperation bekräftigten. Dass der Trump-Schock nicht zu noch deutlicheren Absatzbewegungen von den USA führte, hatte wiederum innenpolitische Gründe. In Deutschland etwa fehlten dazu die notwendigen Mehrheiten in Öffentlichkeit und Parlament, die Kosten einer europäischen Autonomie zu schultern - nicht nur, aber auch im Bereich der Verteidigungsausgaben. Folgerichtig kalkulierten die östlichen Bündnispartner, dass eine Gewährleistung der kollektiven Verteidigung ohne die USA unmöglich wäre. Diese Regierungen zielten daher statt auf Hedging-Strategien vielmehr darauf, den US-Präsidenten persönlich zu besänftigen und gingen ansonsten die riskante Wette ein, dass die Wahlen 2020 einen transatlantischeren Präsidenten ins Amt befördern würden.

\section{Back to the Future? Biden und die Zukunft der transatlantischen Beziehungen}

Mit dem Wahlsieg Bidens ging diese Wette zumindest vorläufig auf, denn der neue Präsident bemühte sich schnell darum, die Bündnistreue der USA gegenüber den Alliierten in Europa (und Asien) zu unterstreichen. Symbolische Politik und Rhetorik sind wichtige Elemente politsicher Praxis, um verlorengegangenes Vertrauen wiederaufzubauen. Auch konkrete Maßnahmen wie die Rückkehr zum Pariser Klimaabkommen oder die Verlängerung des New START-Abrüstungsabkommens mit Russland zielen auf die Wiederherstellung von Erwartungssicherheit in der Außenpolitik. Zudem zeigt das Beispiel der Beilegung des langjährigen deutsch-amerikanischen Streits über Nord Stream 2, dass die transatlantischen Partner trotz unterschiedlicher Interessen zur Kompromissfindung fähig sind.

Allerdings verbleiben strukturelle Gründe, die auch in Zukunft Potenzial für transatlantische Krisen liefern. Blickt man auf die innergesellschaftliche Debatte in den USA, dann wird klar, dass sich am grundsätzlichen Fokus auf innenpolitische Problemlagen seit dem Beginn der Obama-Präsidentschaft wenig geändert hat. Auch Bidens Priorität liegt auf wirtschafts- und sozialpolitischen Reformprojekten, wie dem Infrastrukturpaket. Außenpolitische Entscheidungen werden daher vor allem an innergesellschaftlichen Präferenzen ausgerichtet. Die Abzugsentscheidung aus Afghanistan ist hierfür ein Beispiel. Bidens Begründung konzentrierte sich in erster Linie auf den nationalen Kontext - nämlich die Kriegsmüdigkeit der US-Öffentlich- 
keit und die Frage nach den nationalen Interessen der USA. ${ }^{2}$ Um diesen Rechnung zu tragen, nahm der Präsident auch eine Beschädigung der internationalen Glaubwürdigkeit der USA in Kauf, während die Interessen der Verbündeten kaum eine Rolle zu spielen schienen.

Die Sensitivität der Wählerschaft hinsichtlich der Kosten und Nutzen der Führungsrolle der USA führt dazu, dass die US-Administration ihren strategischen Fokus enger fasst und sich zunehmend auf die Einhegung des einzigen hegemonialen Herausforderers, China, konzentriert. Diese auch von Republikanern in seltener Überparteilichkeit mitgetragene Doktrin hat Konsequenzen für Europa. Sie kann, wie im Falle der neuen Allianz zwischen den USA, Großbritannien und Australien (AUKUS) dazu führen, dass die Interessen europäischer Verbündeter ignoriert werden. Wenig überraschend sprach Präsident Macron angesichts des geplatzten U-Boot-Rüstungsvertrags mit Australien von einem Vertrauensbruch durch die USA (France-24 2021), die Frankreich als Lieferanten ersetzten.

Die Analyse der häufigen Konflikte und selteneren Krisen in den transatlantischen Beziehungen nach 9/11 lässt abschließend die These zu, dass die Resilienz der Sicherheitsgemeinschaft auf offener Kommunikation, der Nutzung etablierter multilateraler Foren zur Konfliktbeilegung und der Berücksichtigung der spezifischen nationalen Interessenlagen beruht.

Funding Open Access funding enabled and organized by Projekt DEAL.

Open Access Dieser Artikel wird unter der Creative Commons Namensnennung 4.0 International Lizenz veröffentlicht, welche die Nutzung, Vervielfältigung, Bearbeitung, Verbreitung und Wiedergabe in jeglichem Medium und Format erlaubt, sofern Sie den/die ursprünglichen Autor(en) und die Quelle ordnungsgemäß nennen, einen Link zur Creative Commons Lizenz beifügen und angeben, ob Änderungen vorgenommen wurden.

Die in diesem Artikel enthaltenen Bilder und sonstiges Drittmaterial unterliegen ebenfalls der genannten Creative Commons Lizenz, sofern sich aus der Abbildungslegende nichts anderes ergibt. Sofern das betreffende Material nicht unter der genannten Creative Commons Lizenz steht und die betreffende Handlung nicht nach gesetzlichen Vorschriften erlaubt ist, ist für die oben aufgeführten Weiterverwendungen des Materials die Einwilligung des jeweiligen Rechteinhabers einzuholen.

Weitere Details zur Lizenz entnehmen Sie bitte der Lizenzinformation auf http://creativecommons.org/ licenses/by/4.0/deed.de.

\section{Literatur}

Böller, Florian, und Steffen Hagemann. 2017. Neuordnung der Sicherheitsgemeinschaft: Obama, die transatlantischen Beziehungen und die Bewältigung sicherheitspolitischer Herausforderungen. Zeitschrift für Außen- und Sicherheitspolitik 10:227-244.

Deutsch, Karl W., Sidney A. Burrell, Robert A. Kann, Maurice Lee, Martin Lichtermann, Raymond E. Lindgren, Francis L. Loewenheim, und Richard W. van Wagenen. 1957. Political community and the north atlantic area. International organizations in the light of historical experience. Princeton: Princeton University Press.

\footnotetext{
2 Biden: „To those asking for a third decade of war in Afghanistan, I ask: What is the vital national interest? In my view, we only have one: to make sure Afghanistan can never be used again to launch an attack on our homeland“ (White House 2021).
} 
Factba.se. 2018. Interview: Tucker Carlson Interviews Donald Trump in Helsinki. https://factba.se/ transcript/donald-trump-interview-tucker-carlson-fox-helsinki-july-17-2018. Zugegriffen: 8. Nov. 2021.

Forsberg, Tuomas. 2005. German foreign policy and the war on Iraq: anti-americanism, pacifism or emancipation? Security Dialogue 36:213-231.

France-24. 2021. Australia must „redefine“ relations after submarine spat „broke“ trust, Macron says. https://www.france24.com/en/europe/20211028-australia-must-redefine-relations-after-submarinespat-broke-trust-macron-says. Zugegriffen: 8. Nov. 2021.

Gates, Robert. 2011. Text of speech by Robert gates on the future of NATO. https://www.atlanticcouncil. org/blogs/natosource/text-of-speech-by-robert-gates-on-the-future-of-nato/. Zugegriffen: 8. Nov. 2021.

Haesebrouck, Tim. 2021. NATO burden sharing after the Wales summit: a generalized set qualitative analysis. Defense and Peace Economics https://doi.org/10.1080/10242694.2021.1928435.

Hansel, Mischa, und Kai Oppermann. 2016. Counterfactual reasoning in foreign policy analysis: the case of German nonparticipation in the Libya intervention of 2011. Foreign Policy Analysis 12:109-127.

Junker, Detlef. 2021. Deutschland und die USA 1871-2021. Heidelberg: HeiBooks.

Koschut, Simon. 2014. Emotional (security) communities: the significance of emotion norms in inter-allied conflict management. Review of International Studies 40:533-558.

Koschut, Simon. 2016. Normative change and security community disintegration: undoing peace. Basingstoke: Palgrave Macmillan.

New York Times. 2016. Transcript: Donald Trump's foreign policy speech. www.nytimes.com/2016/04/ 28/us/politics/transcript-trump-foreign-policy.html.. Zugegriffen: 8. Nov. 2021.

Politbarometer. 2018. Große Mehrheit: USA kein verlässlicher Partner. Forschungsgruppe Wahlen. https:// www.forschungsgruppe.de/Umfragen/Politbarometer/Archiv/Politbarometer_2018/Mai_2018/\#_. Zugegriffen: 8. Nov. 2021.

Reuters. 2019. France's Macron: I'm not sorry I called NATO brain dead. https://www.reuters.com/article/ us-france-nato-braindead-idUSKBN1Y21JE. Zugegriffen: 8. Nov. 2021.

Schuette, Leonard A. 2021. Why NATO survived Trump: the neglected role of secretary-general Stoltenberg. International Affairs 97:1863-1881.

Schünemann, Wolf J., Sebastian Harnisch, und Stefan Artmann. 2018. Cybersicherheit und Rollenwandel. Eine rollentheoretische Diskursanalyse der transatlantischen Cybersicherheitspolitik im Lichte des NSA-Skandals. Zeitschrift für Politikwissenschaft 28:263-287.

Terriff, Terry. 2004. Fear and loathing in NATO: the atlantic alliance after the crisis over Iraq. Perspectives on European Politics and Society 5:419-446.

Tuschhoff, Christian. 2021. From internalization to externalization: the impact of state-society relationsships on US foreign policy. In Hegemonic transition: global economic and security order in the age of trump, Hrsg. Florian Böller, Welf Werner, 21-42. Basingstoke: Palgrave Macmillan.

White House. 2011. Remarks by the president on the way forward in Afghanistan. https://obamawhitehouse. archives.gov/the-press-office/2011/06/22/remarks-president-way-forward-Afghanistan. Zugegriffen: 8 . Nov. 2021.

White House. 2021. Remarks by president Biden on the end of the war in Afghanistan. https://www. whitehouse.gov/briefing-room/speeches-remarks/2021/08/31/remarks-by-president-biden-on-theend-of-the-war-in-afghanistan/. Zugegriffen: 8. Nov. 2021. 